Target characterization using decomposition of the time-reversal operator: electromagnetic scattering from small ellipsoids

D. H. Chambers, J. G. Berryman

May 26, 2006

Inverse Problems 
This document was prepared as an account of work sponsored by an agency of the United States Government. Neither the United States Government nor the University of California nor any of their employees, makes any warranty, express or implied, or assumes any legal liability or responsibility for the accuracy, completeness, or usefulness of any information, apparatus, product, or process disclosed, or represents that its use would not infringe privately owned rights. Reference herein to any specific commercial product, process, or service by trade name, trademark, manufacturer, or otherwise, does not necessarily constitute or imply its endorsement, recommendation, or favoring by the United States Government or the University of California. The views and opinions of authors expressed herein do not necessarily state or reflect those of the United States Government or the University of California, and shall not be used for advertising or product endorsement purposes. 


\title{
Target characterization using decomposition of the time-reversal operator: electromagnetic scattering from small ellipsoids
}

\author{
D. H. Chambers and J. G. Berryman \\ Lawrence Livermore National Laboratory \\ Livermore, CA 94551-9900*
}

(Dated: May 18, 2006)

\begin{abstract}
Decomposition of the time-reversal operator for an array, or equivalently the singular value decomposition of the multistatic response matrix, has been used to improve imaging and localization of targets in complicated media. Typically, each singular value is associated with one scatterer even though it has been shown in several cases that a single scatterer can generate several singular values. In earlier papers Chambers and Berryman $[1,2]$ showed that a small spherical scatterer can generate up to six singular values depending on the array geometry and sphere composition. It was shown that the existence and characteristics of multiple singular values for each scatterer can, in principle, be used to determine certain properties of the scatterers, e.g. conducting or non-conducting material. In this paper, we extend this analysis to non-spherical targets and show how orientation information about the target may be obtained from the spectrum of singular values. The general properties of the decomposition for small non-spherical dielectric (and possibly conductive) targets in an electromagnetic field are derived and detailed results are obtained for the specific cases of non-magnetic and perfectly conducting needles and disks. It is shown that scatterer orientation can be estimated by tracking the singular values of a linear array as it is rotated around its midpoint.
\end{abstract}

PACS numbers:

*Electronic address: chambers2@llnl.gov 


\section{INTRODUCTION}

There has been an increasing interest in applying time-reversal focusing techniques to electromagnetic waves for enhancing communication and imaging through highly scattering media. This interest follows the rapid development of time-reversal techniques in acoustics (see articles [3, 4] for recent reviews). Though application of time-reversal techniques to electromagnetic waves is straightforward in principle, implementation can be significantly more difficult due to the higher frequencies $(\sim \mathrm{GHz})$ used in applications such as communications and radar. Consequently, most proposed implementations of time-reversal for electromagnetic radiation have been as imaging algorithms and other signal processing applications. However, recent demonstrations of time-reversal focusing (Lerosey, et al. [5] and Henty, et al. [6]) and imaging of real data (Shi, et al. [7] and Liu, et al. [8]) have brought time-reversal methods significantly closer to real-world applications.

Time-reversal methods are motivated by the concept of a time-reversal mirror (TRM [3]). This application consists of an array of transceivers that can record and later retransmit any signal it receives. Each element records the wave field incident on the element, reverses the time signal, then retransmits the reversed waveform. The TRM can be considered a broadband implementation of a phase conjugate mirror (PCM). Like a PCM, if the wavefield from a single source is incident on the TRM, the retransmitted wave focuses back onto the source. The added advantage of a TRM is that all frequencies are focused to the same point at the same time, achieving both spatial and temporal focusing. This occurs even in complex media with multiple scattering and dispersion (see [9-11]). Mathematically, the process is described by the time-reversal operator (TRO), which is derived from the multistatic response matrix (MRM) of the array. This matrix can be obtained by transmitting an impulse from a selected element and recording the received waveforms on all the elements. By repeating the transmit operation for all elements, we obtain a matrix of impulse responses for all combinations of transmitter and receiver. The TRO is the temporal autocorrelation of the MRM (or the product of the MRM with its conjugate in the frequency domain). The behavior of the TRM can be analyzed directly from the MRM by applying the singular value decomposition (SVD) in the frequency domain [12-14]. This is the basis for time-reversal imaging methods in complicated media.

To put the present work in proper context, we will now emphasize some significant dif- 
ferences with prior work. In nearly all of the methods of time-reversal imaging of point-like targets, there is the underlying assumption that each singular value of the multistatic response matrix corresponds to a distinguishable point-like target, i.e. there is a one-to-one correspondence between singular values and observable targets making the rank of the MRM equal to the number of targets (see references $[14,15]$ ). Though not essential for deriving time-reversal imaging algorithms, there has been little work until recently (see Ammari et al. $[16,17])$ that assumes multiple singular values per target. The one-to-one correspondence assumption simplifies the mathematical development of the imaging algorithms and is often valid in experiments when one singular value of each target is dominant $[7,8,14]$. The physical interpretation of this "mathematical" point target assumption is that the target simply reradiates a scaled version of the incident field at the target location. The scale factor is the target strength, or cross-section (see [18-20]). In contrast, a more careful description of scattering from small targets, whose size $a$ is much less than a wavelength $\lambda$, would calculate the scattered field to lowest order in powers of $a / \lambda$ from the governing wave equations (acoustic, elastic, electromagnetic, ...).

For acoustical scattering from spheres, the lowest order scattering is order $(a / \lambda)^{3}$ and consists of a monopole (spherically symmetric) whose strength depends on the compressibility contrast of the sphere, and a dipole whose strength depends on the density contrast[21]. Since the dipole is a vector, there are three degrees of freedom associated with it. These modes, combined with the monopole, give four possible singular values for the MRM. For the electromagnetic case, there are six possible singular values for a small sphere, since the incident field can induce both electric and magnetic dipole moments $[1,2]$. For these "physical" point targets, the target strength can be regarded as a vector operator acting on the incident field at the target location. Thus the spectrum of singular values associated with a small target is produced from the lowest order term for the scattered field, and scales uniformly with target size. The number of singular values remains the same, no matter how small the target is. Thus by using the actual scattering physics, we can analyze the behavior of the MRM for "physical" point targets instead of the "mathematical" point targets. For certain special cases, such as acoustic scattering from neutrally buoyant particles or electromagnetic scattering in two dimensions, the scattering produces only one singular value per target. However, these cases are relatively rare and a true physical description would more generally require multiple singular values per target. 
The presence of multiple singular values for point-like targets has been detected experimentally by Minonzio et al. [22], and used by Prada and Thomas [23] to resolve closely spaced targets. Chambers and Gautesen [21] showed how the actual number of singular values associated with a small sphere in acoustics depends on the array geometry and the material composition. By measuring the spectrum of singular values, it is possible, in principle, to classify the target by its material properties (acoustically soft or hard). Similarly, for electromagnetic scattering from a small sphere, it would be possible to assess the conductivity of the sphere material.

In this paper, we extend the analysis of the time-reversal operator for electromagnetic waves to small non-spherical targets, following the approach taken in reference [1]. For these targets, both material properties and orientation affect the spetrum of singular values. We will show that orientation information could, in principle, be extracted by rotating an array and examining the singular values as functions of the rotation angle. Equivalently, one could define a sequence of subarrays and calculate the sequence of singular values. The use of subarrays during array processing is the basis behind a number of algorithms such as ESPRIT [24]. However, subarrays have yet to be used in time-reversal analysis of targets. We show in this paper that subarray approaches have the potential to extract additional information besides target location.

\section{DERIVATION OF THE MULTISTATIC RESPONSE MATRIX}

Consider an array of $N$ short, crossed dipole elements lying in the plane $z=-z_{a}$ (see figure 1), where $z_{a}$ is the distance between the plane and the target. The position of the $n t h$ element is given by the vector $\mathbf{r}_{n}=\left(\xi_{n}, \eta_{n},-z_{a}\right)$. Following Krauss [25], the electric and magnetic fields at the field point $\mathbf{r}$ radiated from the $n t h$ element are given by

$$
\begin{aligned}
\mathbf{H}_{n}^{(i)}\left(\mathbf{R}_{n}\right) & =\frac{i k e^{i k R_{n}}}{4 \pi R_{n}}\left[\hat{\mathbf{R}}_{n} \times\left(d_{x} I_{n}^{x} \hat{\mathbf{e}}_{x}+d_{y} I_{n}^{y} \hat{\mathbf{e}}_{y}\right)\right] \\
\mathbf{E}_{n}^{(i)}\left(\mathbf{R}_{n}\right) & =-\frac{1}{\varepsilon_{0} c} \hat{\mathbf{R}}_{n} \times \mathbf{H}_{n}^{(i)}\left(\mathbf{R}_{n}\right),
\end{aligned}
$$

where $c$ is the speed of light, $k$ is the wave number, $\varepsilon_{0}$ is the electrical permittivity, and $\mathbf{R}_{n}=\mathbf{r}-\mathbf{r}_{n}$. The scalar $R_{n}$ is the magnitude of the vector $\mathbf{R}_{n}$ and $\hat{\mathbf{R}}_{n}$ is the unit vector

in the direction of $\mathbf{R}_{n}$. The crossed dipoles in the array element (lengths $d_{x}$ and $d_{y}$ ) are driven by the currents $I_{n}^{x}$ and $I_{n}^{y}$. The unit vectors in the $x$ and $y$ directions are $\hat{\mathbf{e}}_{x}$ and $\hat{\mathbf{e}}_{y}$ 


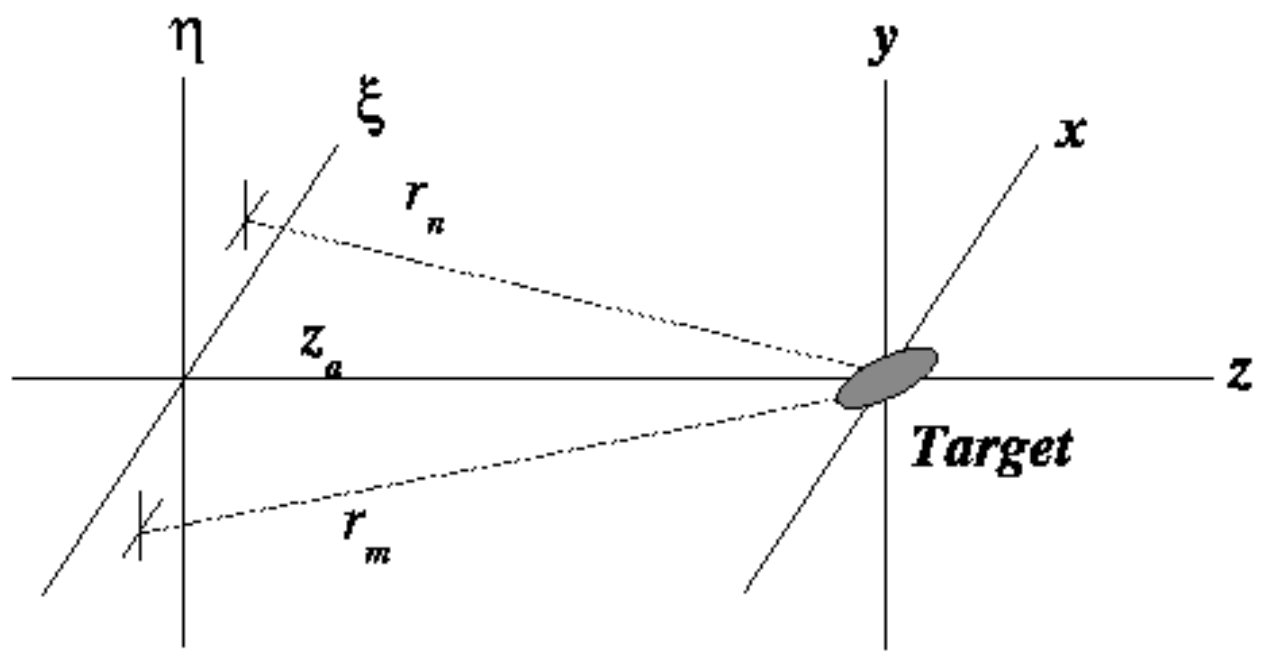

FIG. 1: Geometry of the array and target. Crossed dipole elements $m$ and $n$ are shown at distances $r_{m}$ and $r_{n}$, respectively, from the target. Distance from target to plane of the array is $z_{a}$.

respectively.

Let a target object of characteristic size $a \ll z_{a}$ be placed in front of the array, centered at the origin. The field incident on the target from the $n t h$ element can be approximated as a plane wave coming from the direction of the element. If the target is much smaller than a wavelength, the field scattered from an incident plane wave is given to leading order $\left(\mathcal{O}\left((k a)^{3}\right)\right)[26]$ by

$$
\mathbf{E}^{(s)}(\mathbf{r})=-\frac{k^{2} e^{i k r}}{r}[\hat{\mathbf{r}} \times(\mathbf{m}+\hat{\mathbf{r}} \times \mathbf{p})]
$$

where $\mathbf{p}$ and $\mathbf{m}$ are the induced electric and magnetic dipole moments. These are linearly related to the incident field at the target position $\left(\mathbf{r}=0, \mathbf{R}_{n}=-\mathbf{r}_{n}\right)$ through the electric $\left(\mathbf{D}^{\mathbf{e}}\right)$ and magnetic $\left(\mathbf{D}^{\mathbf{h}}\right)$ dipole tensors:

$$
\mathbf{m}=\frac{1}{\varepsilon_{0} c} \mathbf{D}^{\mathbf{h}} \cdot \mathbf{H}_{n}^{(i)}\left(-\mathbf{r}_{n}\right), \quad \mathbf{p}=\mathbf{D}^{\mathbf{e}} \cdot \mathbf{E}_{n}^{(i)}\left(-\mathbf{r}_{n}\right)
$$

The tensors themselves depend on the shape, size, and electromagnetic material properties (permittivity, permeability, conductivity) of the target. They can be decomposed into their 
principal components,

$$
\mathbf{D}^{\mathbf{e}}=a^{3} \mathbf{U}^{\mathbf{e}} \mathbf{P} \mathbf{U}^{\mathbf{e} T}, \quad \mathbf{D}^{\mathbf{h}}=a^{3} \mathbf{U}^{\mathbf{h}} \mathbf{M} \mathbf{U}^{\mathbf{h} T},
$$

where $\mathbf{P}$ is a diagonal matrix with elements $\left\{p_{1}, p_{2}, p_{3}\right\}, \mathbf{M}$ is a diagonal matrix with elements $\left\{\mu_{1}, \mu_{2}, \mu_{3}\right\}$, and $\mathbf{U}^{\mathbf{e}}$ and $\mathbf{U}^{\mathbf{h}}$ are orthogonal matrices whose columns are the principle directions for their respective tensors. The notation $(\cdot)^{T}$ denotes the transpose of the matrix.

The scattered field induces voltages on each dipole of the array elements. From reference [27], the voltages induced on the dipoles of the $m t h$ element can be expressed as

$$
\begin{aligned}
& V_{m}^{x}=d_{x}\left[\hat{\mathbf{r}}_{m} \times\left(\hat{\mathbf{r}}_{m} \times \hat{\mathbf{e}}_{\mathbf{x}}\right)\right] \cdot \mathbf{E}^{(s)}\left(\mathbf{r}_{m}\right)=-d_{x} \hat{\mathbf{e}}_{\mathbf{x}} \cdot \mathbf{E}^{(s)}\left(\mathbf{r}_{m}\right), \\
& V_{m}^{y}=d_{y}\left[\hat{\mathbf{r}}_{m} \times\left(\hat{\mathbf{r}}_{m} \times \hat{\mathbf{e}}_{\mathbf{y}}\right)\right] \cdot \mathbf{E}^{(s)}\left(\mathbf{r}_{m}\right)=-d_{y} \hat{\mathbf{e}}_{\mathbf{y}} \cdot \mathbf{E}^{(s)}\left(\mathbf{r}_{m}\right),
\end{aligned}
$$

since $\hat{\mathbf{r}}_{m} \cdot \mathbf{E}^{(s)}\left(\mathbf{r}_{m}\right)=0$.

Combining these with the previous expressions for the incident field (1) and scattered field (3), we can calculate the coupling between the voltages $\left\{V_{m}^{x}, V_{m}^{y}\right\}$ in the $m t h$ receiving element and the currents $\left\{I_{n}^{x}, I_{n}^{y}\right\}$ in the $n$th transmitting element generated through scattering from the target (details can be found in ref. [1]):

$$
\left[\begin{array}{c}
V_{m}^{x} \\
V_{m}^{y}
\end{array}\right]=\left[\begin{array}{ll}
K_{m n}^{x x} & K_{m n}^{x y} \\
K_{m n}^{y x} & K_{m n}^{y y}
\end{array}\right]\left[\begin{array}{c}
I_{n}^{x} \\
I_{n}^{y}
\end{array}\right]=\mathbf{K}_{m n}\left[\begin{array}{c}
I_{n}^{x} \\
I_{n}^{y}
\end{array}\right]
$$

The $2 \times 2$ matrix $\mathbf{K}_{m n}$ can be written as

$$
\mathbf{K}_{m n}=\frac{i(k a)^{3} d_{0}^{2}}{4 \pi \varepsilon_{0} c} e^{i k\left(r_{m}+r_{n}\right)} \hat{\mathbf{K}}_{m n}
$$

where $\hat{\mathbf{K}}_{m n}$ is given by

$$
\begin{gathered}
\hat{\mathbf{K}}_{m n}=\frac{1}{r_{m} r_{n}} \sum_{j=1}^{3}\left[\mu_{j} \hat{\mathbf{k}}_{m j}^{\mathbf{h}} \hat{\mathbf{k}}_{n j}^{\mathbf{h} T}-p_{j} \hat{\mathbf{k}}_{m j}^{\mathbf{e}} \hat{\mathbf{k}}_{n j}^{\mathbf{e} T}\right], \\
\hat{\mathbf{k}}_{m j}^{\mathbf{h}}=\left[\begin{array}{l}
\frac{d_{x}}{d_{0}}\left(\hat{r}_{m} \times \hat{e}_{x}\right) \cdot \mathbf{u}_{j}^{\mathbf{h}} \\
\frac{d_{y}}{d_{0}}\left(\hat{r}_{m} \times \hat{e}_{y}\right) \cdot \mathbf{u}_{j}^{\mathbf{h}}
\end{array}\right], \quad \hat{\mathbf{k}}_{m j}^{\mathbf{e}}=\left[\begin{array}{l}
\frac{d_{x}}{d_{0}}\left[\hat{r}_{m} \times\left(\hat{r}_{m} \times \hat{e}_{x}\right)\right] \cdot \mathbf{u}_{j}^{\mathbf{e}} \\
\frac{d_{y}}{d_{0}}\left[\hat{r}_{m} \times\left(\hat{r}_{m} \times \hat{e}_{y}\right)\right] \cdot \mathbf{u}_{j}^{\mathbf{e}}
\end{array}\right],
\end{gathered}
$$

$d_{0}=\sqrt{d_{x}^{2}+d_{y}^{2}}$, and $\mathbf{u}_{j}^{\mathbf{e}}$ and $\mathbf{u}_{j}^{\mathbf{h}}$ are the $j$ th columns of $\mathbf{U}^{\mathbf{e}}$ and $\mathbf{U}^{\mathbf{h}}$. Note that $\hat{\mathbf{K}}_{n m}=\hat{\mathbf{K}}_{m n}^{T}$ by reciprocity. The diagonal elements represent coupling between identical polarizations $(x$ or $y$ ), while the off-diagonal elements represent cross-polarization coupling ( $x$ with $y$ ). 
From this result, the relationship between the transmit currents and the received voltages over the entire array can be constructed. Let $\mathbf{V}$ be the vector of received voltages and $\mathbf{I}$ the vector of transmit currents (both with length $2 N$, accounting for $x$ and $y$ components). Then we can write

$$
\mathbf{V}=\mathbf{T} \mathbf{I}
$$

where

$$
\mathbf{V}=\left[\begin{array}{c}
V_{1}^{H} \\
V_{1}^{V} \\
\vdots \\
V_{m}^{H} \\
V_{m}^{V} \\
\vdots \\
V_{N}^{H} \\
V_{N}^{V}
\end{array}\right], \quad \mathbf{I}=\left[\begin{array}{c}
I_{1}^{H} \\
I_{1}^{V} \\
\vdots \\
I_{n}^{H} \\
I_{n}^{V} \\
\vdots \\
I_{N}^{H} \\
I_{N}^{V}
\end{array}\right],
$$

and the $2 N \times 2 N$ matrix $\mathbf{T}$ is assembled from all the matrices $\mathbf{K}_{m n}$ :

$$
\mathbf{T}=\left[\begin{array}{cccc}
\mathbf{K}_{11} & \mathbf{K}_{12} & \cdots & \mathbf{K}_{1 N} \\
\mathbf{K}_{21} & \mathbf{K}_{22} & \cdots & \mathbf{K}_{2 N} \\
\vdots & \vdots & \ddots & \vdots \\
\mathbf{K}_{N 1} & \mathbf{K}_{N 2} & \cdots & \mathbf{K}_{N N}
\end{array}\right] .
$$

(The current vector $\mathbf{I}$ should not be confused with the identity matrix $\mathfrak{I}$.) The matrix $\mathbf{T}$ is the multistatic response matrix (MRM). It is symmetric and has units of impedance. It can be considered the part of the radiation impedance of the array attributable to the presence of the target. Its $2 N \times 2 N$ size results from the two components of polarization for each element in the array. If only one polarization is used $\left(d_{x}=0\right.$ or $\left.d_{y}=0\right)$, three-quarters of the matrix elements are zero and the useful part of $\mathbf{T}$ reduces to an $N \times N$ matrix. A generalization of this matrix for multiple small targets, and separate transmit and receive arrays can be found in references [16] and [17].

\section{DECOMPOSITION OF THE TIME-REVERSAL OPERATOR}

The time-reversal operator (TRO) is given by $\mathbf{T}^{*} \mathbf{T}$. Its eigenvalues and eigenvectors characterize the properties of the array as a time-reversal system. Each eigenvector specifies 
a set of currents that, when applied to the dipoles in each element of the array, will produce a field that focuses on the target. In addition, the received voltages from the resulting scattered field will be proportional to the conjugate of the applied currents. The proportionality constant is the apparent strength of the scattering mode in the target that is excited by the incident field. Its square is an eigenvalue of the TRO. Rather than decompose $\mathbf{T}^{*} \mathbf{T}$, it is more convenient to calculate the singular value decomposition (SVD) of $\mathbf{T}$. The right singular vectors are identical to the eigenvectors of the TRO, and the squares of the singular values are equal to the eigenvalues. Since $\mathbf{T}$ is symmetric the SVD is [28]

$$
\mathbf{T} \Phi=\Lambda \Phi^{*}
$$

where the singular values $\Lambda$ are real and non-negative. The SVD can be further simplified by factoring out common quantities. Let

$$
\boldsymbol{\Phi}=\frac{1}{\sqrt{i}}\left[\begin{array}{c}
\phi_{1} e^{-i k r_{1}} \\
\phi_{2} e^{-i k r_{1}} \\
\phi_{3} e^{-i k r_{2}} \\
\phi_{4} e^{-i k r_{2}} \\
\vdots \\
\phi_{2 N-1} e^{-i k r_{N}} \\
\phi_{2 N} e^{-i k r_{N}}
\end{array}\right], \quad \Lambda=\frac{(k a)^{3} d_{0}^{2}}{4 \pi \varepsilon_{0} c} \lambda
$$

then the SVD becomes

$$
\hat{\mathbf{T}} \phi=\lambda \phi^{*}
$$

where

$$
\hat{\mathbf{T}}=\left[\begin{array}{cccc}
\hat{\mathbf{K}}_{11} & \hat{\mathbf{K}}_{12} & \cdots & \hat{\mathbf{K}}_{1 N} \\
\hat{\mathbf{K}}_{21} & \hat{\mathbf{K}}_{22} & \cdots & \hat{\mathbf{K}}_{2 N} \\
\vdots & \vdots & \ddots & \vdots \\
\hat{\mathbf{K}}_{N 1} & \hat{\mathbf{K}}_{N 2} & \cdots & \hat{\mathbf{K}}_{N N}
\end{array}\right] .
$$

The quantities factored out of $\Phi$ are phase factors that cause the field created by the singular vectors to focus on the target position. Since there is only one target, this distribution of phases is common to all singular vectors (see [21]). The remaining quantities in $\phi$ represent a complex amplitude distribution that depends more on the scattering pattern of the target than its location. It is reasonable to expect that the amplitude distributions for each singular vector contain useful information about target shape and orientation. 
From the expansion of $\hat{\mathbf{K}}_{m n}$ in equation (9), we see that $\hat{\mathbf{T}}$ can be expressed as a sum of six terms, each of which is an outer product of vectors:

$$
\hat{\mathbf{T}}=\sum_{j=1}^{3}\left[-p_{j} \mathbf{g}_{j} \mathbf{g}_{j}^{T}+\mu_{j} \mathbf{g}_{j+3} \mathbf{g}_{j+3}^{T}\right],
$$

where

$$
\mathbf{g}_{j}=\left[\begin{array}{c}
\frac{1}{r_{1}} \hat{\mathbf{k}}_{1 j}^{\mathbf{e}} \\
\vdots \\
\frac{1}{r_{N}} \hat{\mathbf{k}}_{N j}^{\mathbf{e}}
\end{array}\right], \quad \mathbf{g}_{j+3}=\left[\begin{array}{c}
\frac{1}{r_{1}} \hat{\mathbf{k}}_{1 j}^{\mathbf{h}} \\
\vdots \\
\frac{1}{r_{N}} \hat{\mathbf{k}}_{N j}^{\mathbf{h}}
\end{array}\right], \quad j=1,2,3 .
$$

The singular vectors for matrices of this form can be expressed as linear combinations of the vectors $\mathbf{g}_{1}$ through $\mathbf{g}_{6}$,

$$
\phi=\sum_{j=1}^{6} \gamma_{j} \mathbf{g}_{j},
$$

which reduces the SVD for the $2 N \times 2 N$ matrix $\hat{\mathrm{T}}$ to an $\mathrm{SVD}$ of a $6 \times 6$ matrix:

$$
\mathbf{C ~ G} \gamma=\lambda \gamma^{*},
$$

where $G_{j l}=\mathbf{g}_{j}^{T} \mathbf{g}_{l}$ and $\mathbf{C}=\operatorname{diag}\left(-p_{1},-p_{2},-p_{3}, \mu_{1}, \mu_{2}, \mu_{3}\right)$. Thus the rank of the multistatic response matrix is determined by the physics of the scattering contained in equation (3). This behavior is described by the induced electric and dipole moment vectors, which in three dimensions represent six degrees of freedom. The rank may be less than six in certain special cases, e.g. non-magnetic targets $\left(\mu_{j}=0\right)$. For higher frequencies (or larger targets), additional multipole moments will contribute to the scattered field, increasing the rank of the multistatic response matrix. A similar analysis for the acoustic (scalar) case can be found in references [21, 22, 28].

To summarize, we have shown that the SVD of the original $2 N \times 2 N$ multistatic response matrix reduces to the SVD of a $6 \times 6$ matrix, CG. The physics of the scattering process, not the details of the array, governs the rank of the response matrix. Since we are interested in how information about the target is encoded in the SVD, it would be helpful to determine a way of separating target information from the effects of the array configuration in the decomposition. This has already been partially accomplished in the form of the matrix in equation (21), which is a product of the diagonal matrix $\mathbf{C}$ (which specifies the strength of the induced dipole moments) and the matrix $\mathbf{G}$. The latter contains the effects of both array configuration and target orientation (through $\mathbf{U}^{\mathbf{e}}$ and $\mathbf{U}^{\mathrm{h}}$ ). It would be useful to find 
a way to separate these two contributions to $\mathbf{G}$. Careful examination of the structure of $\mathbf{G}$ reveals that each $3 \times 3$ quadrant of the matrix can be decomposed into products of $\mathbf{U}^{\mathbf{e}}$ and $\mathbf{U}^{\mathbf{h}}$ with matrices that depend only on the array configuration:

$$
\mathbf{G}=\left[\begin{array}{cc}
\mathbf{U}^{\mathbf{e} T} \mathbf{B}^{\mathbf{U}} \mathbf{U}^{\mathbf{e}} & \mathbf{U}^{\mathbf{e} T} \mathbf{B}^{\mathbf{X}} \mathbf{U}^{\mathbf{h}} \\
\left(\mathbf{U}^{\mathbf{e} T} \mathbf{B}^{\mathbf{X}} \mathbf{U}^{\mathbf{h}}\right)^{T} & \mathbf{U}^{\mathbf{h} T} \mathbf{B}^{\mathbf{L}} \mathbf{U}^{\mathbf{h}}
\end{array}\right],
$$

where $\mathbf{B}^{\mathbf{U}}, \mathbf{B}^{\mathbf{X}}$, and $\mathbf{B}^{\mathbf{L}}$ are $3 \times 3$ array configuration matrices. These matrices depend only on the array configuration and target location, and are given by

$$
\begin{gathered}
\mathbf{B}^{\mathbf{U}}=\frac{1}{d_{0}^{2}} \sum_{n=1}^{N} \frac{1}{r_{n}^{6}}\left\{d_{x}^{2}\left[\mathbf{r}_{n} \times\left(\mathbf{r}_{n} \times \hat{\mathbf{e}}_{x}\right)\right]^{2}+d_{y}^{2}\left[\mathbf{r}_{n} \times\left(\mathbf{r}_{n} \times \hat{\mathbf{e}}_{y}\right)\right]^{2}\right\} \\
\mathbf{B}^{\mathbf{X}}=\frac{1}{d_{0}^{2}} \sum_{n=1}^{N} \frac{1}{r_{n}^{5}}\left\{d_{x}^{2}\left[\mathbf{r}_{n} \times\left(\mathbf{r}_{n} \times \hat{\mathbf{e}}_{x}\right)\right] \otimes\left[\mathbf{r}_{n} \times \hat{\mathbf{e}}_{x}\right]+d_{y}^{2}\left[\mathbf{r}_{n} \times\left(\mathbf{r}_{n} \times \hat{\mathbf{e}}_{y}\right)\right] \otimes\left[\mathbf{r}_{n} \times \hat{\mathbf{e}}_{y}\right]\right\} \\
\mathbf{B}^{\mathbf{L}}=\frac{1}{d_{0}^{2}} \sum_{n=1}^{N} \frac{1}{r_{n}^{4}}\left\{d_{x}^{2}\left[\mathbf{r}_{n} \times \hat{\mathbf{e}}_{x}\right]^{2}+d_{y}^{2}\left[\mathbf{r}_{n} \times \hat{\mathbf{e}}_{y}\right]^{2}\right\},
\end{gathered}
$$

where $\otimes$ is the vector outer product and $\mathbf{a}^{2}=\mathbf{a} \otimes \mathbf{a}=\mathbf{a a}^{T}$ when $\mathbf{a}$ is a vector. For a continuous aperture, we can replace the sum over array elements with an integral over the aperture normalized by the aperture area. With this decomposition, we have successfully separated target characteristics (size, orientation, composition) from array characteristics. In the remainder of the paper, we investigate particular cases and show how, in principle, one can obtain target information from the singular values and singular vectors of the multistatic response matrix.

\section{PERFECTLY CONDUCTING NEEDLE}

Consider a spheroid positioned at the origin with its axis of symmetry oriented along the unit vector $\mathbf{b}_{3}$. The length along its axis is $2 a_{3}$, and the radius in the perpendicular direction is $a_{1}$. In the limit of $a_{1} / a_{3} \rightarrow 0$, the spheroid becomes a needle. If the needle is perfectly conducting, the leading order contribution to the scattering is given by the electric dipole moment tensor [26]:

$$
\mathbf{D}^{\mathbf{e}}=\frac{a_{3}^{3}}{3 \ln \left(a_{3} / a_{1}\right)} \mathbf{b}_{3}^{T} \mathbf{b}_{3}
$$


Comparing this with the general tensor decompositions in equation (5), we have $\mu_{1}=\mu_{2}=$ $\mu_{3}=0, p_{1}=p_{2}=0, p_{3}=1 / 3$, and define

$$
a^{3}=\frac{a_{3}^{3}}{\ln \left(a_{3} / a_{1}\right)} .
$$

The matrix $\mathbf{U}^{\mathbf{e}}$ becomes the single vector $\mathbf{b}_{3}$, so that $\mathbf{G}=\mathbf{G}^{\mathbf{U}}=\mathbf{b}_{3} \mathbf{H}^{\mathbf{U}} \mathbf{b}_{3}^{T}$ (scalar). Thus the response matrix is rank one and the singular value is

$$
\lambda=\frac{1}{3} \mathbf{b}_{3} \mathbf{H}^{\mathrm{U}} \mathbf{b}_{3}^{T} .
$$

Let $\theta$ be the angle between the needle axis and the $z$ axis $(0 \leq \theta \leq \pi / 2)$, and $\phi$ be the angle between the $x$ axis and the projection of the needle in the $\xi \eta$ plane $(-\pi \leq \phi<\pi)$. Then $\mathbf{b}_{3}=(\cos \phi \sin \theta, \sin \phi \sin \theta, \cos \theta)$, and the singular value for a continuous circular aperture is

$$
\begin{aligned}
\lambda & =\frac{1}{6}\left[\beta_{1}+\beta_{2}+\frac{d_{x}^{2}-d_{y}^{2}}{d_{0}^{2}}\left(\beta_{1}-\beta_{2}\right) \cos (2 \phi)\right] \sin ^{2} \theta+\beta_{3} \cos ^{2} \theta \\
& \sim \frac{1}{6 z_{a}^{2}}\left\{\left[1+\frac{d_{x}^{2}-d_{y}^{2}}{d_{0}^{2}} \cos (2 \phi)\right] \sin ^{2} \theta+O\left(R^{2} / z_{a}^{2}\right)\right\}, R / z_{a} \ll 1 .
\end{aligned}
$$

For the continuous linear array oriented parallel to the $x$ axis, we have

$$
\begin{aligned}
\lambda & =\frac{1}{6}\left[\frac{\alpha_{1} d_{x}^{2}+\alpha_{2} d_{y}^{2}}{d_{0}^{2}}+\frac{\alpha_{1} d_{x}^{2}-\alpha_{2} d_{y}^{2}}{d_{0}^{2}} \cos (2 \phi)\right] \sin ^{2} \theta+\frac{d_{x}^{2}}{d_{0}^{2}} \alpha_{3} \cos ^{2} \theta \\
& \sim \frac{1}{6 z_{a}^{2}}\left\{\left[1+\frac{d_{x}^{2}-d_{y}^{2}}{d_{0}^{2}} \cos (2 \phi)\right] \sin ^{2} \theta+O\left(R^{2} / z_{a}^{2}\right)\right\}, L / z_{a} \ll 1 .
\end{aligned}
$$

Expressions for the $\alpha$ and $\beta$ parameters are found in the Appendix. The asymptotic expressions in equations (27) and (28) are valid in the far field of the array. As expected, the singular values do not depend on $\phi$ when the needle is aligned with the $z$ axis $(\theta=0)$. This result is also found when the $x$ and $y$ dipoles in each element of the circular array are identical $\left(d_{x}=d_{y}\right)$. Similarly, the linear array loses sensitivity to $\phi$ in the far field when $d_{x}=d_{y}$. Conversely, the arrays are maximally sensitive to $\phi$ when there is only one dipole in each array element, i.e. $d_{x}=0$ or $d_{y}=0$ (or when only half the degrees of freedom are in use).

Our primary interest in the singular value is how target information, orientation in this case, is encoded in the singular value. More specifically, can we estimate the orientation of the needle directly from the singular value? Recall that experimental determination of the 
singular value is obtained by applying the SVD directly to the measured $2 N \times 2 N$ response matrix. If the rank of this matrix is one, we might infer that the target is a perfectly conducting needle. Various imaging techniques could be used to locate the target when the medium is free space. Once the target location is estimated, the array could be turned so that the $z$-axis (axis of symmetry) of the array goes through the target. The final step would be determining the orientation.

One method of extracting orientation information would be to compute the singular value using only the $x$ elements $\left(d_{y}=0\right)$, and compare it to the singular value using only the $y$ elements $\left(d_{x}=0\right)$. The difference of singular values for the circular array is proportional to $\cos (2 \phi) \sin ^{2} \theta$. The same is true for the linear array when the needle is in the far field. If the difference is zero, then $\theta=0$ or $\phi=n \pi / 4, n \in\{ \pm 1, \pm 3\}$. This provides some information on target orientation. However, more information may be obtained by relocating the array.

Consider rotating the linear array by an angle $\phi^{\prime}$ in the $\xi \eta$ plane. Not only does this change the orientation of the array, it also changes the orientation of the dipoles in each array element. This rotation is equivalent to rotating the needle by the angle $-\phi^{\prime}$, so that $\phi$ becomes $\phi-\phi^{\prime}$ in the expression for the singular value:

$$
\lambda=\frac{1}{6}\left[\frac{\alpha_{1} d_{x}^{2}+\alpha_{2} d_{y}^{2}}{d_{0}^{2}}+\frac{\alpha_{1} d_{x}^{2}-\alpha_{2} d_{y}^{2}}{d_{0}^{2}} \cos \left(2\left(\phi-\phi^{\prime}\right)\right)\right] \sin ^{2} \theta+\frac{d_{x}^{2}}{d_{0}^{2}} \alpha_{3} \cos ^{2} \theta
$$

It is apparent that $\lambda$ will attain an extremum when $\left|\cos \left(2\left(\phi-\phi^{\prime}\right)\right)\right|=1$. This occurs when $\phi-\phi^{\prime}=n \pi / 2$, i.e. the array is either parallel or perpendicular to the projection of the needle axis in the $\xi \eta$ plane depending on the sign of $\alpha_{1} d_{x}^{2}-\alpha_{2} d_{y}^{2}$. By using only the $x$ dipoles in each element $\left(d_{y}=0\right)$, the extrema of the singular value occur when the array is parallel to the projection of the needle. This approach allows us to estimate the $\phi$ orientation up to an ambiguity of $\pm \pi$. In addition, we could apply the same analysis using the circular array by performing the SVD on the response matrices of a carefully selected sequence of subarrays. Each subarray would consist of the elements of a circular array along a line through the array center. The lines are parameterized by their angles $\phi^{\prime}$ from the $\xi$ axis. By applying the SVD to the sequence of subarrays in order of increasing angle $\phi^{\prime}$, we can replicate the effect of rotating the linear array. ${ }^{1}$

\footnotetext{
1 To strictly reproduce the effect of rotating a linear array, we must also take into account the rotation of the $d_{x}$ and $d_{y}$ dipoles in each array element. This is easily accomplished by applying the rotation matrix generated by $\phi^{\prime}$ to the dipole elements.
} 


\section{PERFECTLY CONDUCTING DISK}

Another interesting case is a perfectly conducting disk positioned at the origin. Again, the axis of symmetry of the disk is specified by the vector $\mathbf{b}_{3}$. We will also require vectors $\mathbf{b}_{1}$ and $\mathbf{b}_{2}$ to specify the plane of the disk (perpendicular to $\mathbf{b}_{3}$ ). For convenience, we choose $\mathbf{b}_{1}=(\cos \phi \cos \theta, \sin \phi \cos \theta,-\sin \theta)$ and $\mathbf{b}_{2}=(-\sin \phi, \cos \phi, 0)$. The leading order contributions to the scattered field as $a_{3} / a_{1} \rightarrow 0$ are produced by two induced electric dipole moments in the plane of the disk (along $\mathbf{b}_{1}$ and $\mathbf{b}_{2}$ ), and an induced magnetic dipole moment along $\mathbf{b}_{3}$. Thus the electric and magnetic moment tensors are

$$
\mathbf{D}^{\mathbf{e}}=\frac{4 a_{1}^{3}}{3 \pi}\left(\mathbf{b}_{1}^{T} \mathbf{b}_{1}+\mathbf{b}_{2}^{T} \mathbf{b}_{2}\right), \quad \mathbf{D}^{\mathbf{h}}=\frac{2 a_{1}^{3}}{3 \pi} \mathbf{b}_{3}^{T} \mathbf{b}_{3}
$$

where $a_{1}(=a)$ is the radius of the disk. Since there are only three contributions to leading order, the $\mathbf{G}$ matrix reduces to

$$
\mathbf{G}=\left[\begin{array}{ccc}
G_{11}^{U} & G_{12}^{U} & G_{13}^{X} \\
G_{12}^{U} & G_{22}^{U} & G_{23}^{X} \\
G_{13}^{X} & G_{23}^{X} & G_{33}^{L}
\end{array}\right]
$$

where $G_{i j}^{U}=\mathbf{b}_{i} \mathbf{B}^{\mathbf{U}} \mathbf{b}_{j}^{T}, G_{i 3}^{X}=\mathbf{b}_{i} \mathbf{B}^{\mathbf{X}} \mathbf{b}_{3}^{T}$, and $G_{33}^{L}=\mathbf{b}_{3} \mathbf{B}^{\mathbf{L}} \mathbf{b}_{3}^{T}$, for $i, j \in\{1,2\}$. Though it is possible to perform the SVD analytically, this serves little purpose as it is more convenient to calculate a specific numerical example.

Figure 2 shows the three singular values that are obtained from the SVD of CG for a linear array rotating around its center in the $\xi \eta$ plane, $L / z_{a}=1$. If the axis of the disk is oriented along the $z$ axis $(\theta=0)$, there is no dependence on $\phi$, as expected. For $\theta>0$ the first and third singular values are maximum when the array is parallel to the projection of the disk axis in the $\xi \eta$ plane. In contrast, the second singular value is minimum when the array is aligned. Thus we can estimate the $\phi$ orientation up to a $\pm \pi$ ambiguity using any of the singular values. 
1

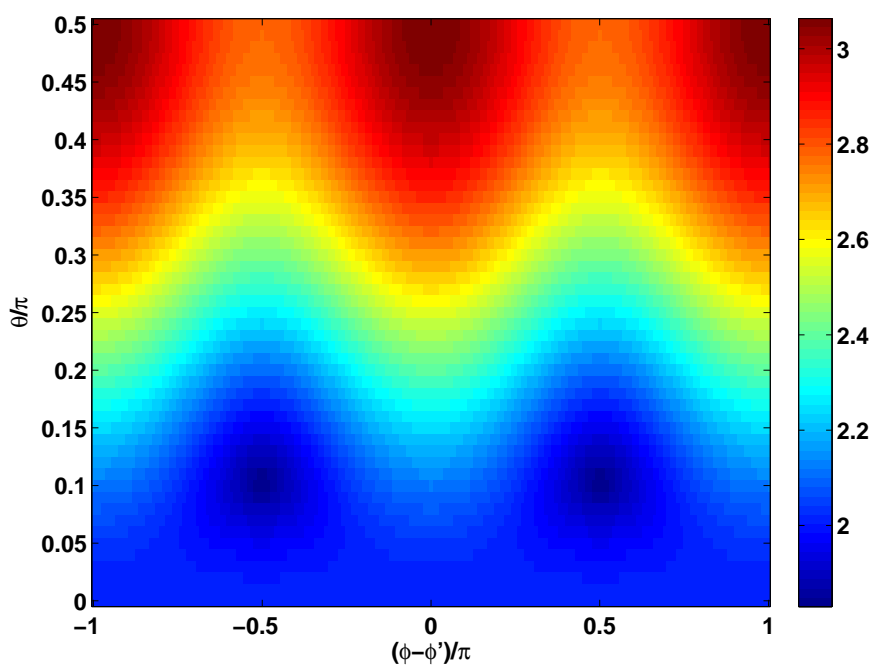

2

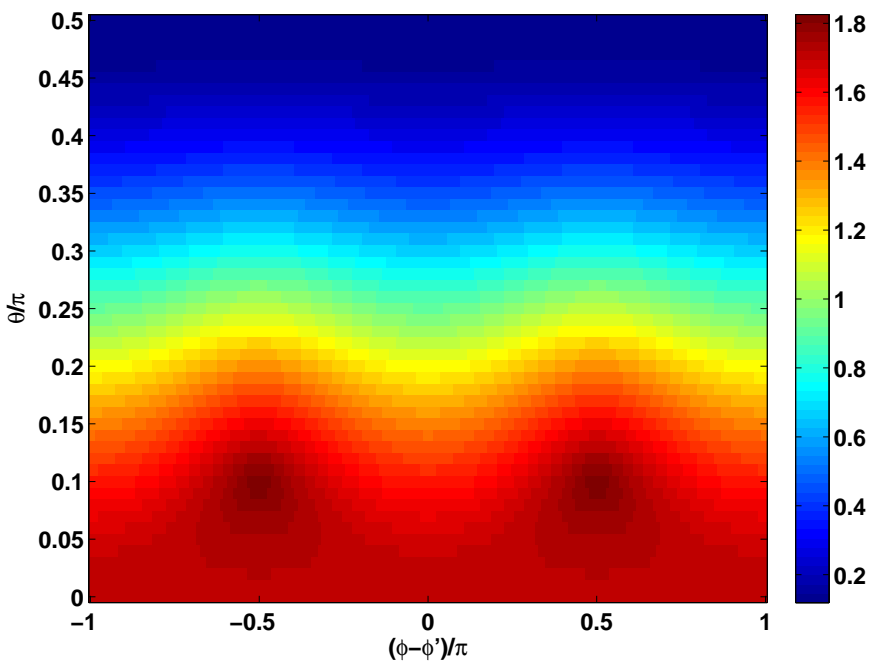

3

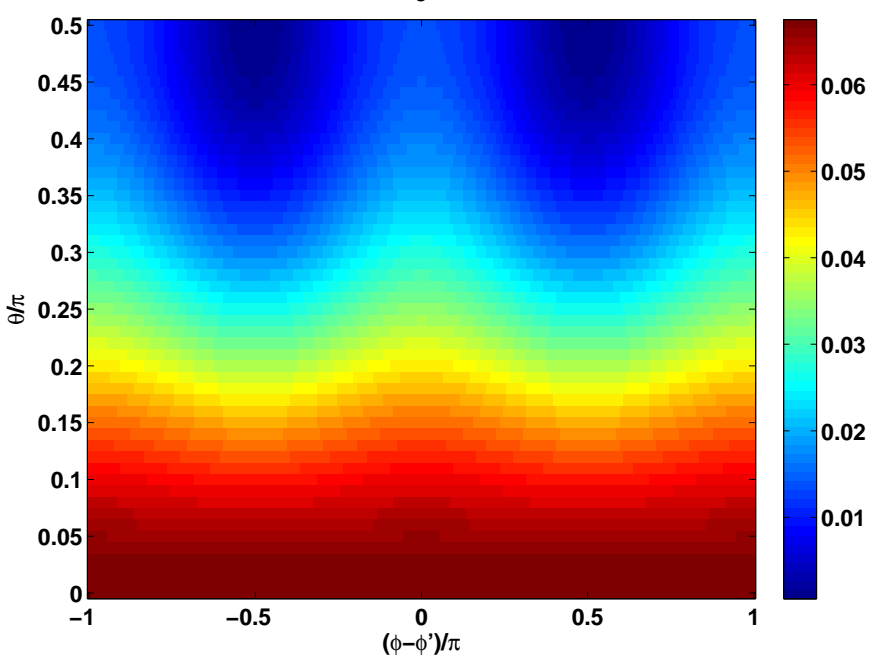

FIG. 2: Three singular values for a rotating linear array $\left(d_{x}=d_{y}\right)$ and a perfectly conducting disk as functions of polar angle $\theta$ and relative rotation angle $\phi-\phi^{\prime}$ at a range $L / z_{a}=1$. 


\section{DIELECTRIC NEEDLE OR DISK}

Finally, we consider the case of a dielectric (non-conducting) needle or disk placed at the origin. There are no induced magnetic moments so $\mathbf{G}=\mathbf{U}^{\mathbf{e} T} \mathbf{B}^{\mathbf{U}} \mathbf{U}^{\mathbf{e}}$, with

$$
\mathbf{U}^{\mathbf{e}}=\left[\begin{array}{lll}
\mathbf{b}_{1}^{T} & \mathbf{b}_{2}^{T} & \mathbf{b}_{3}^{T}
\end{array}\right]
$$

The values of the principal components $p_{1}, p_{2}$, and $p_{3}$ for both needle and disk are shown in Table I. The only difference between the disk and the needle is in the form of $p_{3}$. The overall size scale $a$ is given by $a=\left(a_{1}^{2} a_{3}\right)^{1 / 3}$. Figures 3 and 4 show the three singular values for both the needle and disk for a linear array rotating around its center in the $\xi \eta$ plane, $L / z_{a}=1$. The value of the relative permittivity $\epsilon_{r}$ is 2 . There are only minor differences between the needle and disk. When $\theta>0$, the first singular value has a maximum and the second singular value a minimum when the array is parallel to the projection of the needle or disk axis in the $\xi \eta$ plane. Interestingly, the third singular value shows minimal variation with $\phi$, making it difficult to obtain any orientation information from it. Fortunately, the first two singular values show signficant variation with $\phi$, making it possible to estimate $\phi$ by rotating the array.

TABLE I. Electric dipole strength parameters for a dielectric needle and disk

\begin{tabular}{c|c|c|} 
& Needle & Disk \\
\hline$p_{1}$ & $\frac{\epsilon_{r}-1}{3}$ & $\frac{\epsilon_{r}-1}{3}$ \\
\hline$p_{2}$ & $\frac{\epsilon_{r}-1}{3}$ & $\frac{\epsilon_{r}-1}{3}$ \\
\hline$p_{3}$ & $\frac{2}{3} \frac{\epsilon_{r}-1}{\epsilon_{r}+1}$ & $\frac{\epsilon_{r}-1}{\epsilon_{r}}$ \\
\hline
\end{tabular}




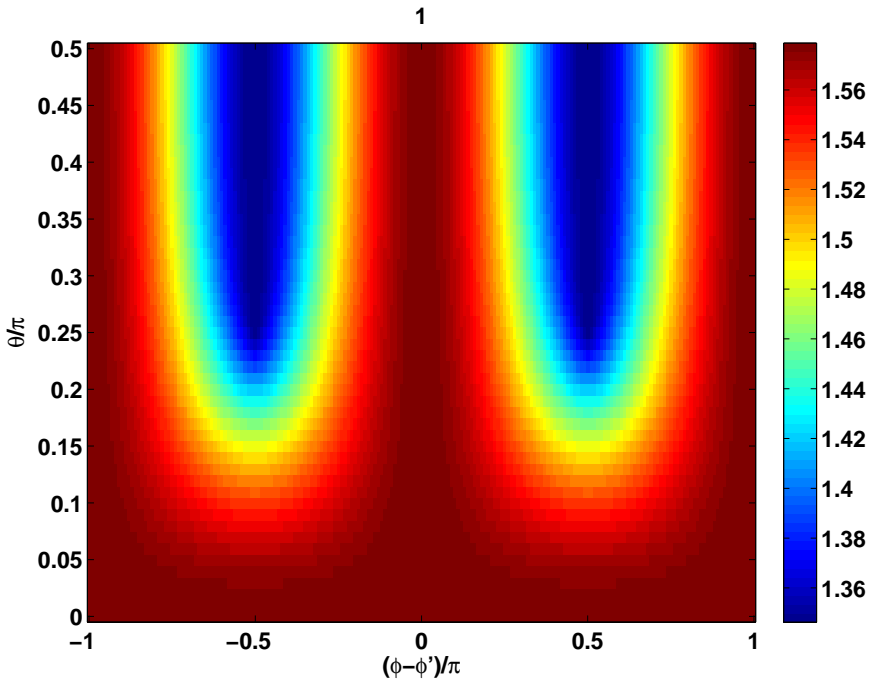

2

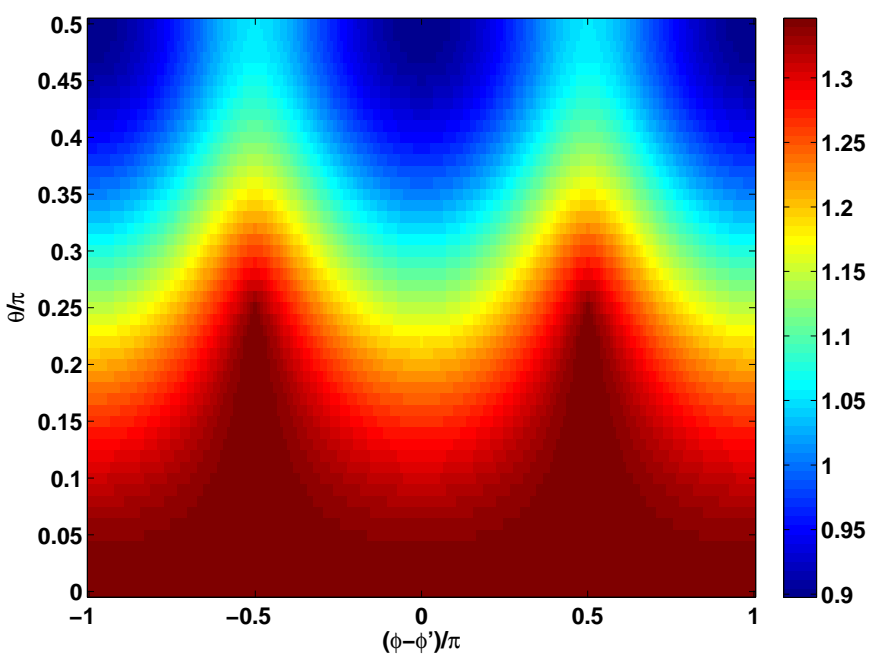

3

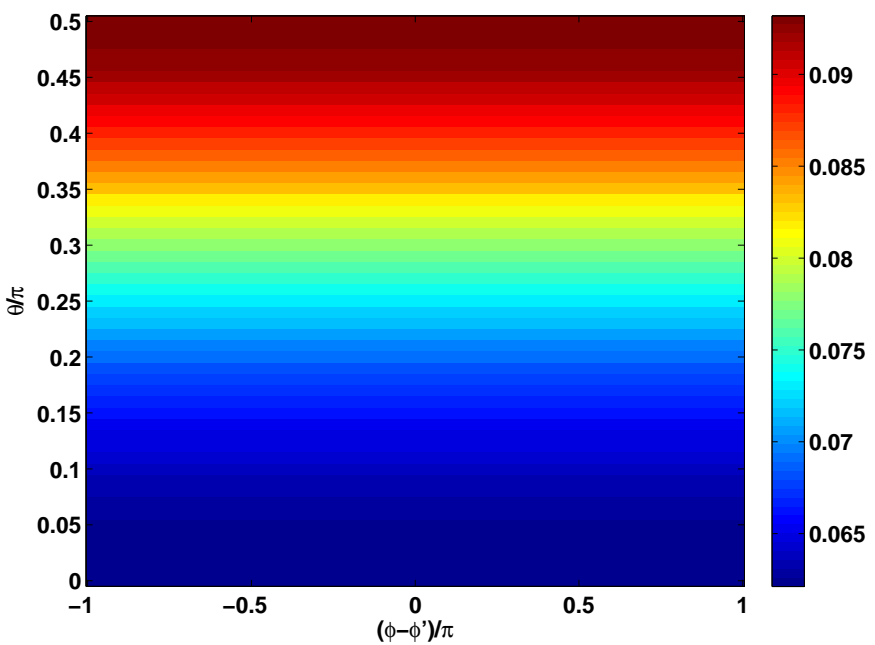

FIG. 3: Three singular values for a rotating linear array $\left(d_{x}=d_{y}\right)$ and a dielectric needle as functions of polar angle $\theta$ and relative rotation angle $\phi-\phi^{\prime}$ at a range $L / z_{a}=1$. 

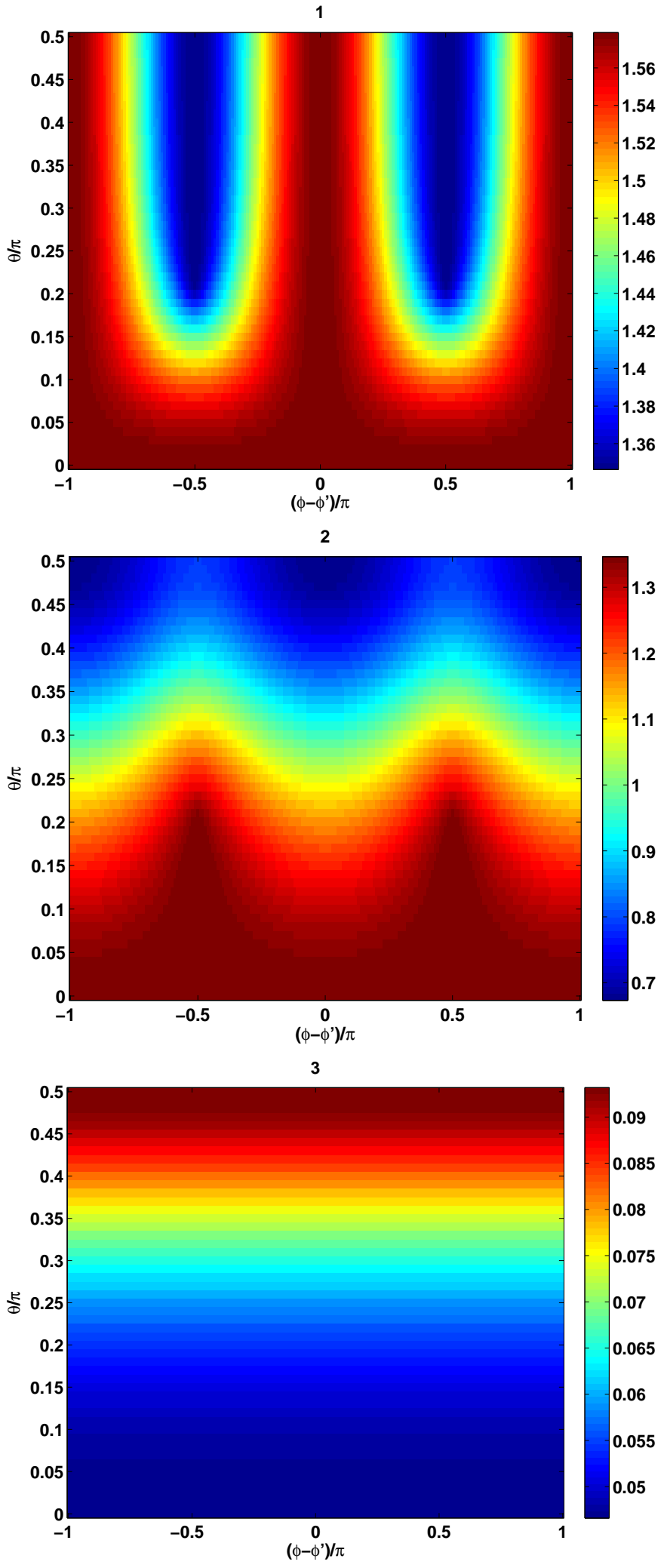

FIG. 4: Three singular values for a rotating linear array $\left(d_{x}=d_{y}\right)$ and a dielectric disk as functions of polar angle $\theta$ and relative rotation angle $\phi-\phi^{\prime}$ at a range $L / z_{a}=1$. 


\section{SUMMARY AND CONCLUSION}

We have generalized the decomposition of the TRO for planar arrays found in references $[1,2]$ to the case of small non-spherical targets. We have shown that the rank of the multistatic response matrix is determined by the number of principal components in the electric and magnetic moment tensors. The array configuration defines a mapping between the moment tensor spaces and the space of the TRO. The presence of multiple singular values for a given target is a property of the nature of the scattering operator at lowest order in $a / \lambda$ and persists in the limit of $a / \lambda \rightarrow 0$. Thus this property is a characteristic of actual "physical" point targets, as opposed to the "mathematical" point targets commonly assumed in the development of time-reversal imaging algorithms.

We show that orientation information can, both in principle and in practice, be extracted from observing the behavior of the singular values of the MRM as one rotates a linear array. This rotation need not be physical, but could be accomplished by applying the SVD to a sequence of properly defined subarrays in a larger circular or other planar array. Though we have shown this for only a few examples, it suggests that a substantial amount of target information could be extracted by applying the SVD to carefully defined sequences of subarrays. This would extend the utility of the time-reversal imaging methods found in references $[8,15-18]$ to include target characterization.

\section{Appendix A: Expressions for Array Configuration Matrix Elements.}

Here we derive the matrix elements for $\mathbf{B}^{\mathbf{U}}, \mathbf{B}^{\mathbf{X}}$, and $\mathbf{B}^{\mathbf{L}}$ for the special cases of continuous circular and linear arrays when the target is at the origin, which is a position along an axis

of symmetry. Since the arrays are considered continuous, we replace the summations in equation (23) with integrations over the aperture of the array, normalized by the array aperture (or length):

$$
\begin{aligned}
& \mathbf{B}^{\mathbf{U}}=\frac{1}{d_{0}^{2} A} \int_{A} \frac{1}{r^{6}}\left\{d_{x}^{2}\left[\mathbf{r} \times\left(\mathbf{r} \times \hat{\mathbf{e}}_{x}\right)\right]^{2}+d_{y}^{2}\left[\mathbf{r} \times\left(\mathbf{r} \times \hat{\mathbf{e}}_{y}\right)\right]^{2}\right\} d A \\
& \mathbf{B}^{\mathbf{X}}=\frac{1}{d_{0}^{2} A} \int_{A} \frac{1}{r^{5}}\left\{d_{x}^{2}\left[\mathbf{r} \times\left(\mathbf{r} \times \hat{\mathbf{e}}_{x}\right)\right] \otimes\left[\mathbf{r} \times \hat{\mathbf{e}}_{x}\right]+d_{y}^{2}\left[\mathbf{r} \times\left(\mathbf{r} \times \hat{\mathbf{e}}_{y}\right)\right] \otimes\left[\mathbf{r} \times \hat{\mathbf{e}}_{y}\right]\right\} d A \\
& \mathbf{B}^{\mathbf{L}}=\frac{1}{d_{0}^{2} A} \int_{A} \frac{1}{r^{4}}\left\{d_{x}^{2}\left[\mathbf{r} \times \hat{\mathbf{e}}_{x}\right]^{2}+d_{y}^{2}\left[\mathbf{r} \times \hat{\mathbf{e}}_{y}\right]^{2}\right\} d A,
\end{aligned}
$$


where $\mathbf{r}=\left(\xi, \eta,-z_{a}\right)$ and $r=\sqrt{\xi^{2}+\eta^{2}+z_{a}^{2}}$. For a circular array the integral becomes

$$
\frac{1}{A} \int_{A}(\cdot) d A \rightarrow \frac{1}{\pi R^{2}} \int_{0}^{R} \int_{-\pi}^{\pi}(\cdot) d \phi \rho d \rho,
$$

where $\rho=\sqrt{\xi^{2}+\eta^{2}}, \tan \phi=\xi / \eta$, while for a linear array aligned with the $x$ axis it is

$$
\left.\frac{1}{A} \int_{A}(\cdot) d A \rightarrow \frac{1}{L} \int_{-L / 2}^{L / 2}(\cdot)\right|_{\eta=0} d \xi .
$$

Using the above formulas, we can calculate the array configuration matrices for a continuous circular array of radius $R$ :

$$
\begin{aligned}
\mathbf{B}^{\mathbf{U}} & =\left[\begin{array}{cccc}
\frac{d_{x}^{2}}{d_{0}^{2}} \beta_{1}+\frac{d_{y}^{2}}{d_{0}^{2}} \beta_{2} & 0 & 0 \\
0 & \frac{d_{x}^{2}}{d_{0}^{2}} \beta_{2}+\frac{d_{y}^{2}}{d_{0}^{2}} \beta_{1} & 0 \\
0 & 0 & \beta_{3}
\end{array}\right], \\
\mathbf{B}^{\mathbf{X}} & =\left[\begin{array}{ccc}
0 & \frac{d_{x}^{2}}{d_{0}^{2}} \beta_{4} & 0 \\
-\frac{d_{y}^{2}}{d_{0}^{2}} \beta_{4} & 0 & 0 \\
0 & 0 & 0
\end{array}\right], \\
\mathbf{B}^{\mathbf{L}} & =\left[\begin{array}{ccc}
\frac{d_{y}^{2}}{d_{0}^{2}} \beta_{5} & 0 & 0 \\
0 & \frac{d_{x}^{2}}{d_{0}^{2}} \beta_{5} & 0 \\
0 & 0 & \beta_{6}
\end{array}\right],
\end{aligned}
$$

where

$$
\begin{aligned}
& \beta_{1}=\frac{7 R^{2}+10 z_{a}^{2}}{16\left(R^{2}+z_{a}^{2}\right)^{2}}+\frac{3}{8 R^{2}} \ln \left(1+\frac{R^{2}}{z_{a}^{2}}\right), \\
& \beta_{2}=-\frac{3 R^{2}+2 z_{a}^{2}}{16\left(R^{2}+z_{a}^{2}\right)^{2}}+\frac{1}{8 R^{2}} \ln \left(1+\frac{R^{2}}{z_{a}^{2}}\right), \\
& \beta_{3}=\frac{R^{2}}{4\left(R^{2}+z_{a}^{2}\right)^{2}} \\
& \beta_{4}=\frac{1}{3 R^{2}}\left[4-\frac{z_{a}\left(3 R^{2}+4 z_{a}^{2}\right)}{\left(R^{2}+z_{a}^{2}\right)^{3 / 2}}\right], \\
& \beta_{5}=\frac{1}{R^{2}+z_{a}^{2}}, \\
& \beta_{6}=-\frac{1}{2\left(R^{2}+z_{a}^{2}\right)}+\frac{1}{2 R^{2}} \ln \left(1+\frac{R^{2}}{z_{a}^{2}}\right) .
\end{aligned}
$$

In the far field $\left(R / z_{a} \ll 1\right)$,

$$
\begin{aligned}
& \beta_{1} \sim \frac{1}{z_{a}^{2}}\left(1-\frac{R^{2}}{z_{a}^{2}}\right), \quad \beta_{2} \sim \frac{R^{4}}{24 z_{a}^{6}}\left(1-\frac{9 R^{2}}{4 z_{a}^{2}}\right), \quad \beta_{3} \sim \frac{R^{2}}{4 z_{a}^{4}}\left(1-\frac{2 R^{2}}{z_{a}^{2}}\right), \\
& \beta_{4} \sim \frac{1}{z_{a}^{2}}\left(1-\frac{R^{2}}{z_{a}^{2}}\right), \quad \beta_{5} \sim \frac{1}{z_{a}^{2}}\left(1-\frac{R^{2}}{z_{a}^{2}}\right), \quad \beta_{6} \sim \frac{R^{2}}{z_{a}^{4}}\left(1-\frac{4 R^{2}}{3 z_{a}^{2}}\right) .
\end{aligned}
$$


For a continuous linear array of length $L$ oriented parallel to the $x$ axis, the configuration matrices are:

$$
\begin{aligned}
\mathbf{B}^{\mathbf{U}} & =\left[\begin{array}{ccc}
\frac{d_{x}^{2}}{d_{0}^{2}} \alpha_{1} & 0 & 0 \\
0 & \frac{d_{y}^{2}}{d_{0}^{2}} \alpha_{2} & 0 \\
0 & 0 & \frac{d_{x}^{2}}{d_{0}^{2}} \alpha_{3}
\end{array}\right], \\
\mathbf{B}^{\mathbf{X}} & =\left[\begin{array}{ccc}
0 & \frac{d_{x}^{2}}{d_{0}^{2}} \alpha_{4} & 0 \\
\frac{d_{y}^{2}}{d_{0}^{2}} \alpha_{5} & 0 & 0 \\
0 & 0 & 0
\end{array}\right], \\
\mathbf{B}^{\mathbf{L}} & =\left[\begin{array}{ccc}
\frac{d_{y}^{2}}{d_{0}^{2}} \alpha_{6} & 0 & 0 \\
0 & \frac{d_{x}^{2}}{d_{0}^{2}} \alpha_{6} & 0 \\
0 & 0 & \frac{d_{y}^{2}}{d_{0}^{2}} \alpha_{7}
\end{array}\right],
\end{aligned}
$$

where

$$
\begin{aligned}
\alpha_{1} & =\frac{3 L^{2}+20 z_{a}^{2}}{2\left(L^{2}+4 z_{a}^{2}\right)^{2}}+\frac{3}{4 L z_{a}} \arctan \left(\frac{L}{2 z_{a}}\right), \\
\alpha_{2} & =\frac{L^{2}}{2\left(L^{2}+4 z_{a}^{2}\right)^{2}}+\frac{2}{L z_{a}} \arctan \left(\frac{L}{2 z_{a}}\right), \\
\alpha_{3} & =\frac{L^{2}-4 z_{a}^{2}}{2\left(L^{2}+4 z_{a}^{2}\right)^{2}}+\frac{1}{4 L z_{a}} \arctan \left(\frac{L}{2 z_{a}}\right), \\
\alpha_{4} & =\frac{4 L^{2}+24 z_{a}^{2}}{3 z_{a}\left(L^{2}+4 z_{a}^{2}\right)^{3 / 2}}, \\
\alpha_{5} & =-\frac{2 L^{2}+8 z_{a}^{2}}{z_{a}\left(L^{2}+4 z_{a}^{2}\right)^{3 / 2}}, \\
\alpha_{6} & =\frac{2}{L^{2}+4 z_{a}^{2}}+\frac{1}{L z_{a}} \arctan \left(\frac{L}{2 z_{a}}\right), \\
\alpha_{7} & =-\frac{2}{L^{2}+4 z_{a}^{2}}+\frac{1}{L z_{a}} \arctan \left(\frac{L}{2 z_{a}}\right) .
\end{aligned}
$$

In the far field $\left(L / z_{a} \ll 1\right)$,

$$
\begin{gathered}
\alpha_{1} \sim \frac{1}{z_{a}^{2}}\left(1-\frac{L^{2}}{4 z_{a}^{2}}\right), \quad \alpha_{2} \sim \frac{1}{z_{a}^{2}}\left(1-\frac{5 L^{2}}{96 z_{a}^{2}}\right), \quad \alpha_{3} \sim \frac{L^{2}}{12 z_{a}^{4}}\left(1-\frac{9 L^{2}}{20 z_{a}^{2}}\right), \\
\alpha_{4} \sim \frac{1}{z_{a}^{2}}\left(1-\frac{5 L^{2}}{24 z_{a}^{2}}\right), \quad \alpha_{5} \sim-\frac{1}{z_{a}^{2}}\left(1-\frac{L^{2}}{8 z_{a}^{2}}\right), \\
\alpha_{6} \sim \frac{L^{2}}{z_{a}^{4}}\left(1-\frac{L^{2}}{6 z_{a}^{2}}\right), \quad \alpha_{7} \sim \frac{L^{2}}{12 z_{a}^{4}}\left(1-\frac{3 L^{2}}{10 z_{a}^{2}}\right) .
\end{gathered}
$$




\section{Acknowledgment}

This work was performed under the auspices of the U. S. Department of Energy by the University of California, Lawrence Livermore National Laboratory under Contract No. W7405-Eng-48. Work supported in part by the DOE Office of Advanced Scientific Computing Research.

[1] D. H. Chambers and J. G. Berryman, IEEE Trans. Ant. Prop. 52, 1729 (2004).

[2] D. H. Chambers and J. G. Berryman, Phys. Rev. Lett. 92, 0239021 (2004).

[3] M. Fink, D. Cassereau, A. Derode, C. Prada, P. Roux, M. Tanter, J.-L. Thomas, and F. Wu, Rep. Prog. Phys. 63, 1933 (2000).

[4] M. Fink and C. Prada, Inverse Probl. 17, R1 (2001).

[5] G. Lerosey, J. de Rosny, A. Tourin, A. Derode, G. Montaldo, and M. Fink, Phys. Rev. Lett. 92, $1939041(2004)$.

[6] B. E. Henty and D. D. Stancil, Phys. Rev. Lett. 93, 2439041 (2004).

[7] G. Shi and A. Nehorai, Comm. Inform. Sys. 5, 227 (2005).

[8] D. Liu, G. Kang, L. Li, Y. Chen, S. Vasudevan, W. Joines, Q. Liu, J. Krolik, and L. Carin, IEEE Trans. Ant. Prop. 53, 3058 (2005).

[9] A. Tourin, M. Fink, , and A. Derode, Waves Random Media 10, R31 (2000).

[10] P. Roux, B. Roman, and M. Fink, Appl. Phys. Lett. 70, 1811 (1997).

[11] P. Roux and M. Fink, J. Acoust. Soc. Am. 107, 2418 (2000).

[12] C. Prada and M. Fink, Wave Motion 20, 151 (1994).

[13] C. Prada, J.-L. Thomas, and M. Fink, J. Acoust. Soc. Am. 97, 62 (1995).

[14] C. Prada, S. Manneville, D. Spoliansky, and M. Fink, J. Acoust. Soc. Am. 109, 2067 (1996).

[15] L. Borcea, G. Papanicolaou, C. Tsogka, and J. Berryman, Inverse Problems 18, 1247 (2002).

[16] H. Ammari, E. Iakovleva, and D. Lesselier, SIAM J. Sci. Comp. 27, 130 (2005).

[17] H. Ammari, E. Iakovleva, D. Lesselier, and G. Perrusson, SIAM J. Sci. Comp. ??, ?? (2006).

[18] A. J. Devaney, IEEE Trans. Ant. Prop. 53, 1600 (2005).

[19] S. K. Lehman and A. J. Devaney, J. Acoust. Soc. Am. 113, 2742 (2003).

[20] F. K. Gruber, E. A. Marengo, and A. J. Devaney, J. Acoust. Soc. Am. 115, 3042 (2004). 
[21] D. H. Chambers and A. K. Gautesen, J. Acoust. Soc. Am. 109, 2616 (2001).

[22] J.-G. Minonzio, C. Prada, D. H. Chambers, D. Clorennec, and M. Fink, J. Acoust. Soc. Am. 117, 789 (2005).

[23] C. Prada and J.-L. Thomas, J. Acoust. Soc. Am. 114, 235 (2003).

[24] R. Roy and T. Kailath, IEEE Trans. Acoust., Speech, Signal Process. ASSP-37, 984 (1989).

[25] J. D. Kraus, Antennas (McGraw-Hill, San Francisco, 1988), 2nd ed.

[26] G. Dassios and R. Kleinman, Low Frequency Scattering (Clarendon Press, Oxford, 2000).

[27] W. L. Stutzman and G. A. Thiele, Antenna Theory and Design (John Wiley and Sons, New York, 1981).

[28] D. H. Chambers, J. Acoust. Soc. Am. 112, 411 (2002). 